\title{
PLASMA-BASED LASER PULSE CONTROL TECHNIQUES FOR LASER WAKEFIELD ACCELERATORS*
}

\author{
R. F. Hubbard, ' P. Sprangle, T. G. Jones, C. I. Moore, A. Ting, \\ Plasma Physics Division, Naval Research Laboratory, Washington, DC \\ B. Hafizi, Icarus Research, Inc., Bethesda, MD \\ D. Kaganovich, J. R. Peñano, LET Corp., Washington, DC \\ D. Gordon, NRC/NRL Research Associate \\ T. M. Antonsen, Jr., University of Maryland \\ A. Zigler, Hebrew University, Jerusalem, Israel \\ P. Mora, École Polytechnique, Palaiseau, France
}

\begin{abstract}
Possible plasma-based techniques for controlling or modifying the laser pulse in a laser wakefield accelerator (LWFA) are examined, using laser propagation simulation codes. Laser pulse shortening or chopping prior to injection into a plasma guiding channel occurs when the peak laser power is slightly above the critical power for relativistic focusing. A channel-guided LWFA may undergo further compression due to relativistic modulation effects. Multi-stage capillary discharges allow the channel density to be extended and tapered for optimal acceleration in future LWFA experiments.
\end{abstract}

\section{INTRODUCTION}

Future laser wakefield accelerator (LWFA) experiments will require precise control of the laser pulse shape, spot size, and phase slippage rate. This paper utilizes laser simulation codes to examine several plasma-based techniques for controlling laser pulse parameters. For example, pulse shortening prior to injection into the plasma channel and main acceleration region may be achieved by propagating the pulse in a short, high density plasma region where the laser power slightly exceeds the critical power for nonlinear focusing [1]. Relativistic modulation may further compress the pulse as it propagates in the plasma channel [2]. Simulations and analytical scaling models [3-5] predict that acceleration to energies above $1 \mathrm{GeV}$ is possible with currently-available lasers, but this will probably require long plasma channels with axially-varying plasma densities. Multi-stage capillary discharges may provide a method for extending plasma channel length and generating the desired axial density profiles.

The simulations have been performed using the quasistatic propagation codes LEM [6] and WAKE [7]. The models make similar assumptions and have similar features except that LEM uses a cold fluid model for the plasma while WAKE employs a particle simulation model.

\footnotetext{
*Supported by the Department of Energy, the Office of Naval Research, and the US-Israeli Binational Science Foundation

"hubb@ppdmail.nrl.navy.mil
}

\section{BACKGROUND}

Short laser pulses propagating in plasmas exhibit a variety of linear and nonlinear phenomena. In vacuum, the characteristic length for expansion due to diffraction is the Rayleigh length $Z_{R}=\pi r_{L}^{2} / \lambda$, where $r_{L}$ and $\lambda$ are the laser spot size and wavelength. Guiding or focusing of the pulse occurs from the change in the index of refraction: $\Delta \eta_{\mathrm{p}}=-\omega_{\mathrm{p}}^{2} / 2 \omega^{2}$, where $\omega_{\mathrm{p}}$ and $\omega$ are the plasma and optical frequencies. Relativistic effects can focus or guide a beam when the laser power exceeds the critical focusing power $P_{r}=0.0174\left(\lambda_{p} / \lambda\right)[\mathrm{TW}]$, where $\lambda_{p}=2 \pi c / \omega_{p}$ is the plasma wavelength.

A plasma channel with an on-axis density minimum can focus or guide a laser pulse over many Rayleigh lengths, as has been demonstrated in numerous experiments [8-10] and simulations [3-6,9]. A laser with a Gaussian radial profile propagating in a parabolic channel with plasma density of the form $n(r)=n_{0}+\Delta n\left(r / r_{c}\right)^{2}$ will be guided at an equilibrium radius $r_{M}=\left(r_{c}^{2} / \pi r_{e} \Delta n\right)^{1 / 4}$. Here $n_{0}$ is the onaxis density, $r$ is the distance from the laser pulse axis, $\Delta n$ is the channel depth, $r_{c}$ is the channel radius, and $r_{e}=$ $2.8 \times 10^{-13} \mathrm{~cm}$ is the classical electron radius.

Studies of possible future channel-guided LWFA accelerators [3-5] have concentrated on the standard or resonant regime where the FWHM (full-width half maximum) pulse length $c \tau_{L} \sim \lambda_{p} / 2$. Typical design have peak power $P_{0} \sim 10-30 \mathrm{TW}, \tau_{L} \sim 50-500 \mathrm{fs}, r_{M} \sim 20-60 \mu \mathrm{m}$, and $n_{0}$ chosen to match the $c \tau_{L} \sim \lambda_{p} / 2$ soft resonance condition for optimum acceleration.

\section{RELATIVISTIC PULSE COMPRESSION IN PLASMA CHANNELS}

Intense laser pulses propagating in plasmas also produce instabilities, such as the forward Raman and relativistic modulational instabilities. Pulses with $c \tau_{L} \gg$ $\lambda_{p}$, and $P_{o}>P_{r}$ can be self-guided over several Rayleigh lengths and exhibit strong Raman-driven modulation [6,11]. When $c \tau_{L} \ll \lambda_{p}$, Raman and relativistic modulational instability terms cancel [3], and the pulse may propagate stably over long distances [3-5]. For $c \tau_{L} \sim$ $\lambda_{p}$, the cancellation is incomplete, and the frequency chirp introduced by the relativistic modulational instability 
causes the group velocity to be higher in the back of the pulse than the front, causing energy to flow to the center of the pulse. This suggests the possibility of compressing the pulse in a plasma channel in the regime where $P_{d} / P_{r}<$ 1 , but the intensity is high enough to cause rapid modulational instability growth.

An example of this pulse compression is shown in Fig. 1 , which plots the normalized laser intensity $a^{2}(r, \zeta)$ after $4.8 \mathrm{~cm}$ of propagation in a plasma channel. The results are from a LEM simulation with initial peak normalized intensity $a_{0}^{2}=0.5, n_{0}=1.24 \times 10^{18} \mathrm{~cm}^{-3}, \lambda=0.8 \mu \mathrm{m}, \tau_{L}=80$ fs, $r_{M}=21 \mu \mathrm{m}, P_{0}=7.5 \mathrm{TW}$, and $P_{r}=24.5 \mathrm{TW}$. The coordinate $\zeta=z-c t$ represents the axial position of the pulse, with the original head of the pulse located to the right at $\zeta=0$. The normalized complex laser amplitude or potential $a(r, \zeta)$ in LEM assumes that the fast time scale laser potential $a_{f}=\operatorname{aexp}(i k \zeta)$ plus its complex conjugate, where $k=2 \pi / \lambda$. After propagating $4.8 \mathrm{~cm}$ in the channel, the maximum normalized intensity has increased to 3 , and the pulse width has shrunk to $\sim 15$ fs. The peak power has increased to $27 \mathrm{TW}$, so that most of the compression has been in the axial direction. This technique may be of limited use for LWFA applications because the required propagation distance exceeds the dephasing length [3-5]. However, as discussed in [4], the effect can result in a modest increase in the accelerating electric field as the beam propagates and thus provide a noticeable increase in performance.

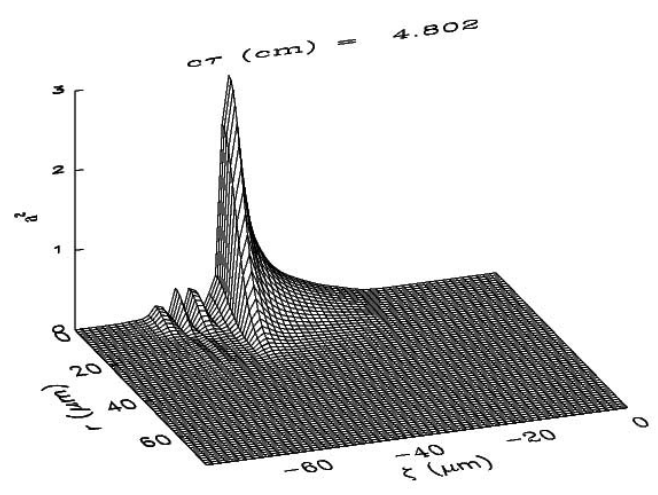

Figure 1. Normalized intensity $a^{2}$ versus $r$ and $\zeta=z-c t$ after propagating $4.8 \mathrm{~cm}$ in a plasma channel from the LEM simulation described above.

\section{RELATIVISTIC-DIFFRACTIVE PULSE SHORTENING AND FOCUSING}

When the laser power exceeds $P_{r}$, relativistic corrections to $\eta(r)$ may overcome diffraction and focus or guide the pulse. A recent analysis by Hafizi, et al., [12] demonstrates that the additional focusing provided by pondermotive expulsion of plasma electrons strongly enhances this effect in the regime where $P \approx P_{r}$. In this regime, the fluid model in LEM often fails, so that WAKE is a more appropriate simulation model. WAKE simulations confirm the importance of including the pondermotive channeling effects retained in Ref. [12] and generally agree well with the envelope equation model. The focusing behavior is a very sensitive function of $P / P_{r}$ in this regime, particularly when the initial laser spot size $r_{0}$ is relatively large.

\subsection{Pulse Shortening Concept and Simulations}

If a laser pulse is injected into a uniform plasma or gas jet with peak power $P_{0}$ slightly above $P_{r}$, then the center of the pulse will be strongly focused while the front and back of the pulse will expand due to diffraction. At the focal point for the peak power pulse center, the intensity will be substantially higher than at injection, and the onaxis intensity will have a substantially shorter pulse width. For $P_{0} \gg P_{r}$, the focusing effect is very strong, and a thin plasma may act as a focusing lens. This regime has recently been analyzed by Ren, et al [13].

Figure 2 plots the normalized on-axis intensity $a^{2}(r=0, \tau)$ at three propagation distances from a WAKE simulation with $a_{0}^{2}=0.332, n_{0}=1.0 \times 10^{19} \mathrm{~cm}^{-3}, \lambda=1.0 \mu \mathrm{m}, \tau_{L}=150$ fs, $r_{0}=15 \mu \mathrm{m}, P_{0}=1.94 \mathrm{TW}$, and $P_{d} / P_{r}=1.0$. The coordinate $\tau=t-z / c$ denotes the axial position in the pulse, with the pulse head to the left at $\tau=0$. The Rayleigh length $Z_{R}=0.0707 \mathrm{~cm}$. At $z / Z_{R}=1.5$, the pulse is approaching the focus, with the on-axis intensity a factor of 4 above its original. At $z / Z_{R}=3.0$, which is near the focal point for the center of the pulse, the peak normalized intensity is more than a factor of 10 above its initial value, and the FWHM width of the intensity profile is reduced from $150 \mathrm{fs}$ to $38 \mathrm{fs}$. The additional oscillations in the tail of the pulse are due to forward Raman instability. A similar simulation with $r_{0}=30 \mu \mathrm{m}$, exhibits a factor of 60 increase in peak intensity at the focus.

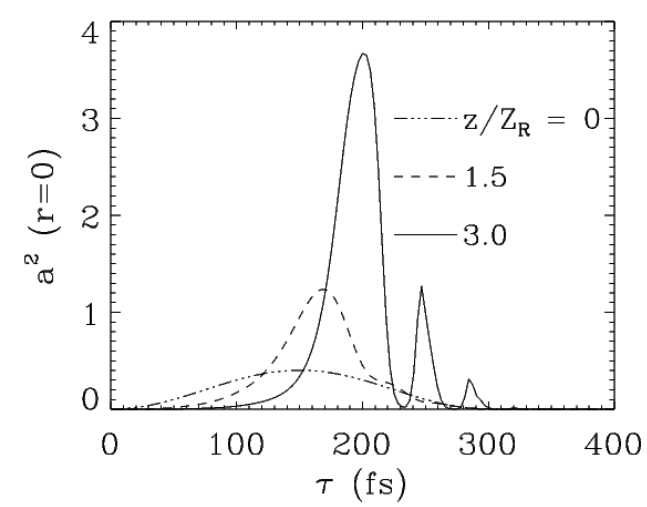

Figure 2 . Normalized on-axis intensity $a^{2}(r=0)$ versus $\tau=t$ $-z / c$ at three different propagation distances from the WAKE simulation described above.

\subsection{Two-stage Channel-guided LWFA}

The pulse shortening effect exhibited in Fig. 2 may be useful for conducting future LWFA experiments on the recently-upgraded $400 \mathrm{fs}^{3}$ (Tabletop Terawatt) laser at the Naval Research Laboratory. The long laser pulse requires a low plasma channel density $\left(\sim 2 \times 10^{16} \mathrm{~cm}^{-3}\right)$ in order to be in the standard LWFA regime. Such channels are difficult to make, and peak accelerating gradients are 
limited to $\sim 1 \mathrm{GV} / \mathrm{m}$ for $\mathrm{T}^{3}$ upgrade parameters [4,5]. It is desirable to have an inexpensive method of shortening the pulse to $\sim 100$ fs prior to injection into the main accelerating channel. However, the matched spot size for typical channel parameters in this regime is $\sim 30 \mu \mathrm{m}$. The uniform plasma simulations described in the previous section produce much smaller spot sizes at the focus, so relativistic-diffractive pulse shortening in a uniform plasma is not appropriate for LWFA applications.

One possible solution is to propagate the pulse in a higher density injection and pulse shortening stage with a weak inverse plasma channel that has an on-axis density maximum and $P_{\delta} / P_{r} \sim 1$. The inverse plasma channel will counter the strong radial collapse of the pulse, while the front and back of the pulse will again expand due to diffraction, resulting in a shorter laser pulse. It is possible that this first stage will also produce accelerated electrons from the background plasma since it is in the regime of a self-modulated LWFA, so that the first stage also functions as an injector. A conventional plasma channel with a density chosen to optimize acceleration $\left(c \tau_{L} \sim \lambda_{p} / 2\right)$ could then be used as the second stage of a two-stage LWFA.

Figure 3 shows the results of a LEM simulation at the end of the pulse shortening stage at $z=0.95 \mathrm{~cm}$. Parameters for the first stage were $a_{0}^{2}=0.578, n_{0}=$ $2.5 \times 10^{18} \mathrm{~cm}^{-3}, \lambda=1.0 \mu \mathrm{m}, \tau_{L}=400 \mathrm{fs}, r_{0}=25 \mu \mathrm{m}, P_{0}=7.8$ TW, $P_{d} / P_{r}=1.1, r_{c}=70 \mu \mathrm{m}$, and $n\left(r_{c}\right) / n_{0}=0.95$. The pulse has a FWHM width of less than $30 \mu \mathrm{m}(100 \mathrm{fs})$, as desired. The trailing oscillations or modulations in the pulse are again due to Raman instability.

The second stage in the simulation is a conventional plasma channel with $n_{0}=2 \times 10^{17} \mathrm{~cm}^{-3}$, and $n\left(r_{c}\right)=4 n_{0}$. The channel recaptures a potion of the laser pulse that had diffracted away from the axis. At $z=1.6 \mathrm{~cm}$, the FWHM width of the pulse has increased to $170 \mathrm{fs}$, and the peak accelerating field is $6 \mathrm{GV} / \mathrm{m}$. The pulse thus remains (marginally) in the standard LWFA regime and represents a significant improvement in accelerating gradient over a typical $400 \mathrm{fs}$ case.

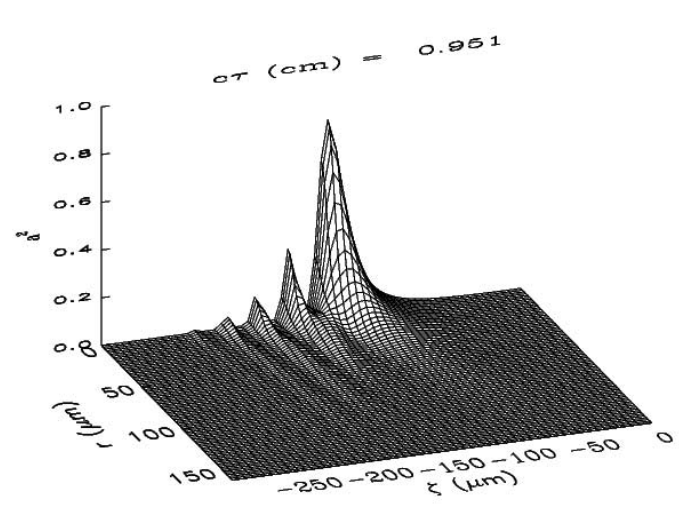

Figure 3. Normalized intensity $a^{2}(r, \zeta)$ after propagating $0.95 \mathrm{~cm}$ for the LEM simulation described above. This is the first stage of a two-stage LWFA.

\section{SEGMENTED CAPILLARY DISCHARGES}

Another technique for improving LWFA performance is to optimize the axial variation of the plasma density in the plasma channel. Injecting the laser into a plasma with increasing plasma density causes the phase of accelerated electrons relative to the wakefield to remain relatively constant. Simulations show that the energy gain may be significantly higher than the usual dephasing limits [3].

Kaganovich, et al., [14] have shown experimentally that the plasma density in an ablative wall capillary discharge may be controlled by varying the discharge voltage. This in principle allows the axial density profile to be optimized by using a series of discrete capillary segments with independent voltage control. An additional feature of this approach is that it should permit arbitrarily long plasma channels. The typical GeV LWFA design requires acceleration distances exceeding $10 \mathrm{~cm}$ [3-5].

\section{SUMMARY}

Several plasma-based techniques for shaping and focusing intense laser pulses and optimizing LWFA performance have been described. These include relativistic pulse compression in plasma channels, relativistic-diffractive pulse shortening for focusing and for two-stage LWFAs, and segmented capillary discharges for controlling and extending the axial plasma density.

\section{REFERENCES}

[1] P. Sprangle, et al., Phys. Rev. Lett. 69, 2200 (1992).

[2] C.J. McKinstrie and R. Bingham, Phys. Fluids B 4, 2626 (1992); P. Sprangle, et al., Phys. Rev. E 61, 4381 (2000).

[3] P. Sprangle, et al., Phys. Rev. Lett. 85, 5110 (2000).

[4] R.F. Hubbard, et al., IEEE Trans. Plasma Sci. 28, 1159 (2000)

[5] R.F. Hubbard, et al., Phys. Rev. E 63, 036502 (2001).

[6] J. Krall, et al., Phys. Rev. E 48, 2157 (1993); Phys. Plasmas 1, 1738 (1994).

[7] T.M. Antonsen and P. Mora, Phys. Fluids B 5, 1440 (1993); P. Mora and T.M. Antonsen, Phys. Plasmas 4, 217 (1997).

[8] C. Durfee and H.M. Milchberg, Phys. Rev. Lett. 71, 2409 (1993); H.M. Milchberg, et al., Phys. Plasmas 3, 2149 (1996).

[9] Y. Ehrlich, et al., Phys. Rev. Lett. 77, 4186 (1996); J. Opt. Soc. Am. B 15, 2416 (1998).

[10] D. Kaganovich, et al., Phys. Rev. E 59, R4769 (1999).

[11] W.B. Mori, IEEE J. Quantum Electron 33, 1942 (1997).

[12] B. Hafizi, et al., Phys. Rev. E 62, 4120 (2000).

[13] C. Ren, et al., Phys. Rev. E 63, 026411 (2001).

[14]D. Kaganovich, et al., Appl. Phys. Lett.78, 3175 (2001). 